\title{
Line Creep in Paper Peeling
}

\author{
J. Koivisto ${ }^{1, \text { a }}$, Xavier Illa ${ }^{1}$, Jari Rosti ${ }^{1}$, and Mikko Alava ${ }^{1}$
}

Aalto University, School of Science and Technology, Department of Applied Physics, 00076 Aalto, Finland

\begin{abstract}
We have studied experimentally the dynamics of the separation of a sheet of paper into two halves in a peeling configuration. The experimental setup consists of a peeling device, where a fracture front is driven along the plane of the paper, with a constant force. The theoretical picture is how an elastic line interacts with a random landscape of fracture toughness. We compare the results with theoretical simulations in several aspects. One recent finding concerns the autocorrelation function of the average front position. The data from the experiments produces so-called cusps or singularities in the correlation function, as predicted by the functional renormalization group theory for elastic lines. Comparisons with simulations with either a short range or a long range elastic kernel demonstrate that the latter agrees with the experimental observations, as expected.
\end{abstract}

\section{Introduction}

An elastic line interacting with a random potential is observed in many physical situations [1] e.g. growth phenomena, domain walls, polymer chains, imbibition or fluid invasion, liquids moving on a solid substrate [2] and fracture fronts. We have recently done a series of experiments on what amounts to paper peeling: a front propagates across the sheet plane as the two halves are separated from each other [3]. The front or line is characterized by a (long-range) elasticity that tries to keep it flat, while the locally varying fracture toughness creates a disordering force.

The setup can be studied in several separate cases starting from constant strain rate conditions to creep (constant load, discussed here [4]). It is analogous to fracture mechanics for a crack that is confined to a plane, for example using a solid-solid interface between two bulks [5]. Or again, one can simply consider weak layer like in snow (avalances) [6]. This is a particular case of the general problem of propagating, rough cracks [7].

More or less in all the fracture cases above the problem reduces to the competition between the disorder and elasticity. In its simplest form, one has three forces: i) A random pinning force that tries to keep the system in its local minimum. ii) An elastic restoring force that tries to minimize the height differences in a surface. iii) A driving force that forces the system to change its state.

On a more practical level, consider a one-dimensional discretized fracture line: elements $x_{i}$ with each issued a height $h\left(x_{i}\right)$ (or a distance from an original configuration). Then, each element is affected by a force

$$
\begin{aligned}
f & =f_{\text {random }}+f_{\text {drive }}+f_{\text {elastic }} \\
f_{\text {elastic }}(x) & =c \sum_{x_{1}} d x_{1} \frac{h(x)-h\left(x_{1}\right)^{2}}{\left|x-x_{1}\right|}
\end{aligned}
$$

\footnotetext{
${ }^{a}$ e-mail: j uha. koi vi sto@kk. fi
} 
where the two first terms correspond to the random pinning and constant driving force respectively. The last term is an elastic term, and scales as $1 / k$ in Fourier space: it arises from non-local elasticity. The integration (sum) limits can be chosen to be periodic (usual theoretical case) or finite (open boundaries). The random pinning force $f_{\text {random }}$ can also be augmented by a contribution from thermal fluctuations, that add on the top of a quenched (function of $h(x)$ only) term. At $T=0$, there is a depinning transition at a critical force (or stress intensity factor) separating a moving line from a frozen one, and the transition is described by a set of exponents appropriate for a second-order non-equilibrium one. The thermal fluctuations become important in the low-velocity regime since then the line velocity attains its value due to thermally-excited local avalanches $[4,8]$.

The various properties of the dynamics, including the "creep formula" or the average line creep velocity, have been studied recently theoretically using simulations and renormalization group arguments. A particularly interesting aspect in this sense is the dynamics of the average position (height $\langle h\rangle$ ). It has been recently realized to be related to the way the interface averages the disorder (fracture toughness here) during its motion: due to the jerky, intermittent dynamics one has "cusps" or discontinuities in the auto-correlation function of the position and in the disorder as well $[2,9]$.

\section{Experiments}

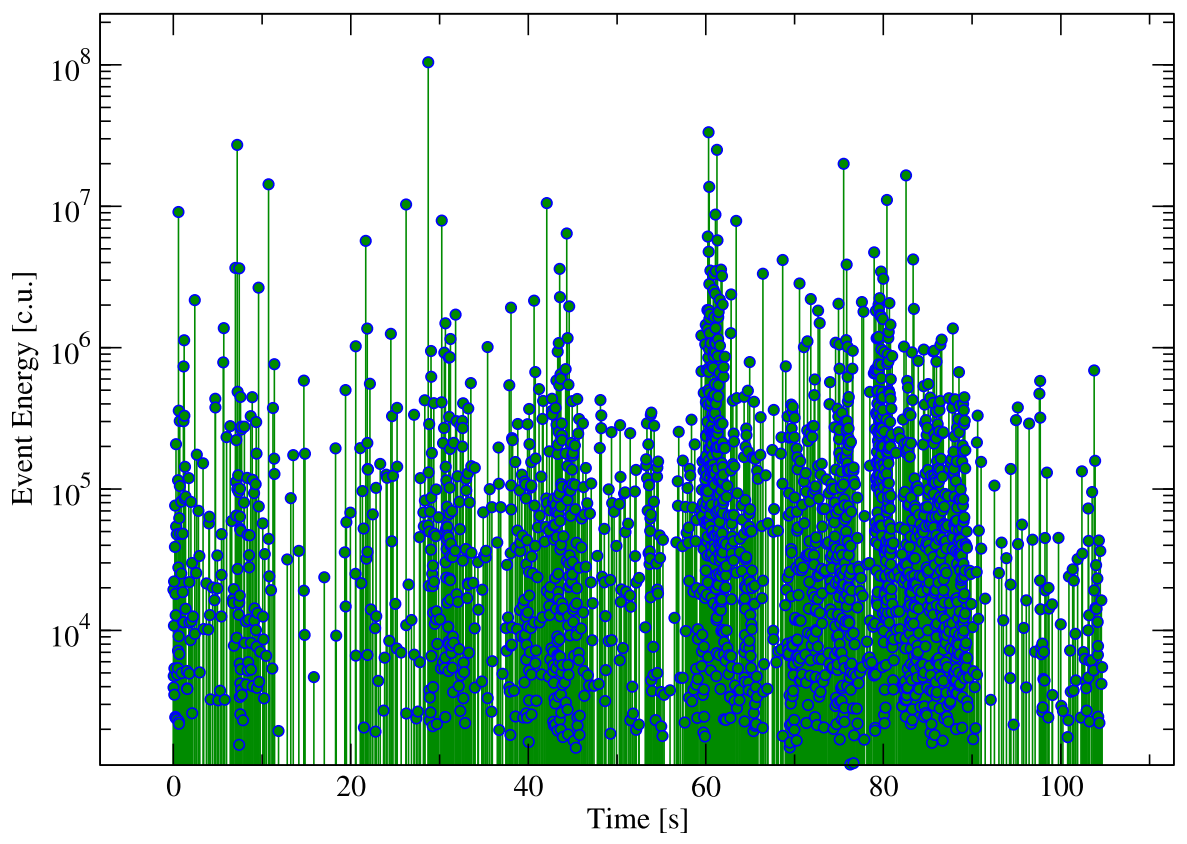

Fig. 1. Acoustic emission event energy time series for a single experiment. The jerky motion of crack propagation is seen, including a clustering of events.

The experimental device is the same used in $[4,10]$. A $30 \mathrm{~mm}$ wide paper sheet is peeled in half along its major plane between two rotating cylinders. This produces roughly a $30 \mathrm{~mm}$ wide fracture 
front which is driven through the sample. The dynamics are intermittent and the driving force is constant.

The average height, $\bar{h}^{\prime}(t)=\left\langle h^{\prime}(x, t)\right\rangle_{x}$ and the acoustic emission (AE) is recorded during the experiment. For $\bar{h}^{\prime}$ an Omron laser distance sensor is used. The AE is recorded using standard plate-like piezo sensor and analog preamplification and filtering. The sample rate for both, AE and $\bar{h}^{\prime}$ is 312.5 $\mathrm{kHz}$, but due to noise the laser data is averaged over 300 scans, giving it a $1 \mathrm{~ms}$ time resolution. The acoustic emission data is processed into $t_{i}, E_{i}$-pairs, where a heuristic method is used to identify the event and its energy. The same method is used in References [3,4,10,11].

Figure 1 shows an example of the $t_{i}, E_{i}$ timeseries for a single creep experiment. There is more than ten thousand events per experiment.

The data analysis relies more on the acoustic emission data as it has far better spatial and temporal resolution. Accuracy in determining event start time, $t_{i}$ is the order of $1 \mu \mathrm{s}$ and multiple events can be recognized even if overlapping.

\section{Comparison to Simulations}

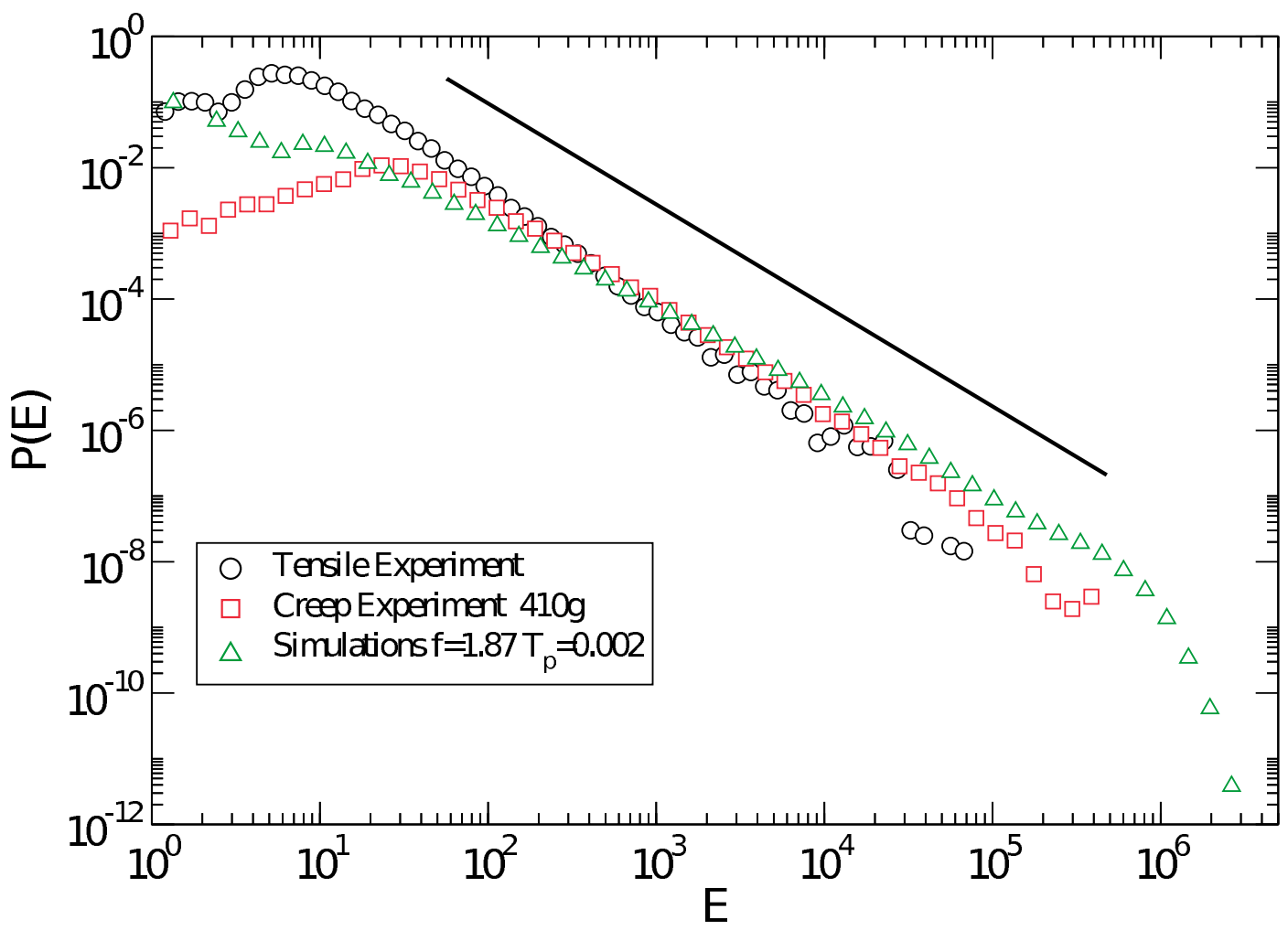

Fig. 2. Probability distribution of event energies. The slope of the line is -1.6. The tensile experiment refers to a similar experiment but with a constant velocity, from [10].

For simulations we use the discrete dynamics with a constant force. A line segment advances one step, if a force threshold is exceeded. A more detailed description for the simulations is available in Reference [10]. Here the focus is purely on the creep experiments. 
Figure 2 shows the comparison between event energy distributions for experiments (creep) and simulations. The experiment labeled as "tensile" is the same experiment but with constant speed [3]. The distributions for waiting time $\tau=t_{i+1}-t_{i}$ and velocity are also similar for all three cases [10].

\section{Creep velocity}

To compare the experiments to existing theory, one can find the dependence between driving mass and velocity. This is also what is Functional renormalization group theory predicts for the velocity near depinning transition in finite temperatures [8]

$$
\mathrm{V}\left(m_{e f f}\right) \sim \exp \left(-\frac{U_{c}}{T} m_{e f f}^{\mu}\right)
$$

Equation (3) is more commonly written with $F$ and $F_{c}$ than the effective mass, $m_{\text {eff }}$. Here, the magnitude of average fracture toughness $\langle T\rangle$, or $\langle m\rangle$ in units of driving mass, is left as an open question. Average fracture toughness in paper in other geometries is found to be less than $50 \%$ of the driving mass here. Note that it does not change the value of $\mu=-1$ as $m_{\text {eff }}=m-\langle m\rangle$, where $\langle m\rangle$ is a constant. Figure 3 depicts the average velocity as a function of effective mass.

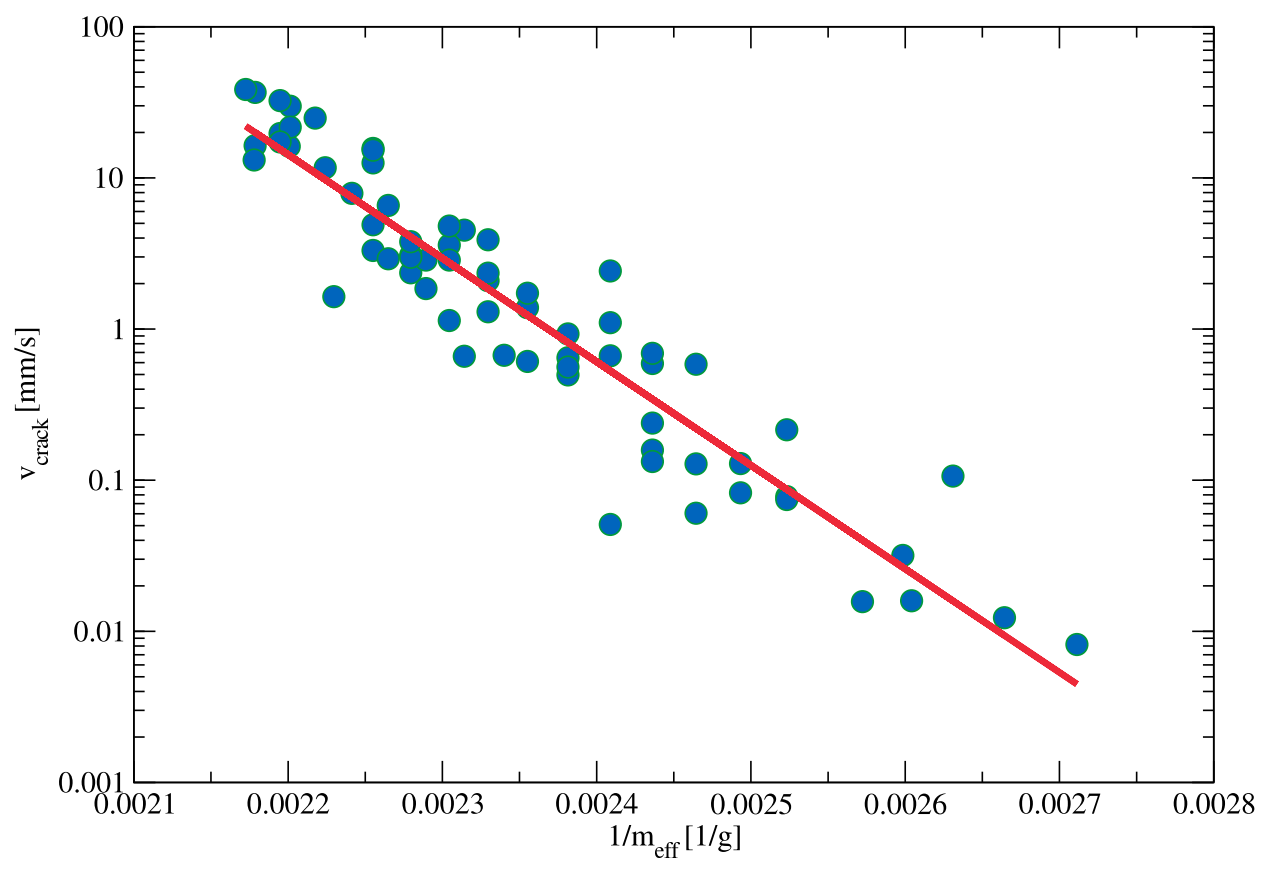

Fig. 3. Average velocity of the fracture front as a function of effective driving force. Line is a fit of equation (3) with $\mu=-1$ [4]. 


\section{Correlation Analysis}

The definitions of quantities follow Ref. [2]. The average interface height $\bar{h}^{\prime}(t)=\left\langle h^{\prime}(x, t)\right\rangle_{x}$, where the average is taken along the $\mathrm{x}$ coordinate perpendicular to propagation direction, is a one dimensional time series. To compare the experiments the height is normalized. First, $h$, is defined as

$$
h(t)=h^{\prime}(t)-\mathrm{V} t-\left\langle h^{\prime}(t)-\mathrm{V} t\right\rangle_{t},
$$

where $v$ is the average propagation velocity for each experiment. Second, the time is scaled to distance with average velocity: $\mathrm{w}=t \mathrm{~V}$. The outcome is the height, $h$, as a function of distance, $\mathrm{w}$ with zero mean and trend.

The normalization allows to compare experiments with different average velocities as the distance, W, changes the material properties more than time. Figure 4 depicts an example of the normalized data.

As a counter argument to above one could say that the affect of load history (time) in paper is highly non-trivial. See for example accelerated creep [12,13], recovery [14] or just a simple creep test [15]. Also the normalization works better for slow experiments.

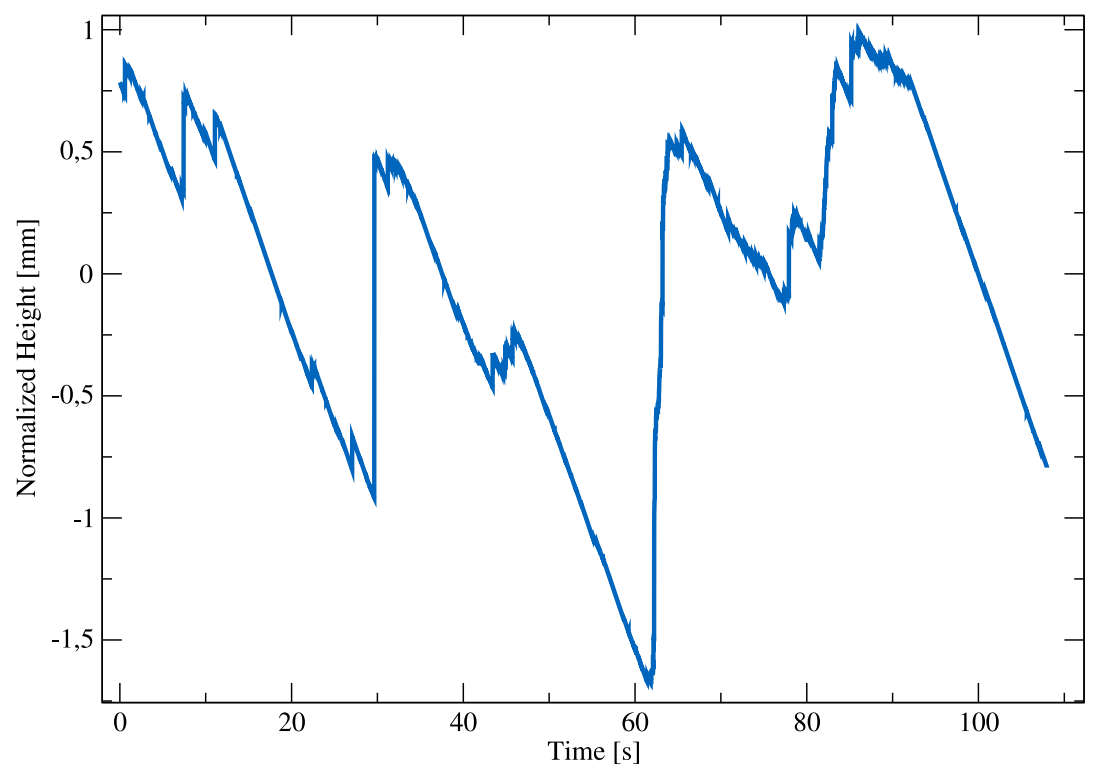

Fig. 4. Fracture front height after normalization, $h(t)$, for a single experiment.

The experimental velocities have large fluctuations due to paper floc size which is order of 1-2 $\mathrm{mm}$, which is much larger than most of the length scales in normalized front height $h$. These fluctuations require more statistics than is available. This issue can be tackled in two ways: i) subtracting a polynomial from the time-series like done in Ref. [2] ii) Drop out the large fluctuations. The latter method is used here.

Both of the above methods have to be done, more or less, by hand. However the latter method can be quantified easily by setting a maximum limit for standard deviation of $h$ during the experiment. Note that this also drops out the experiments with fast average velocity as the fracture front passes a number of flocs in a short time and varies the height rapidly. 
The experimental disorder correlator $\Delta$ for normalized fracture front height is defined as the autocorrelation

$$
\Delta(\mathrm{W}-\mathrm{W})=\langle h(\mathrm{~W}) h(\mathrm{~W})\rangle .
$$

The experiments match with a reasonably good accuracy to the simulations. Figure 5 depicts the normalized disorder correlator for experiments as well as the simulations. For experiments the correlation length is about $0.8 \mathrm{~mm}(\sim 2.5$ c.u. in Fig. 5).

Before the normalization, the short range kernel, behaving as $1 / k^{2}$ in fourier space, differs from the two long range cases with periodic and open boundary conditions[16]. However after the normalization curves overlap with a reasonably good accuracy. Also the experiments agree with the simulations. The sharp cusp near $\mathrm{W}=0$ seen in simulations is rounded a bit in the experiments. The possible reasons for this are the finite temperature as well as the slow speed.

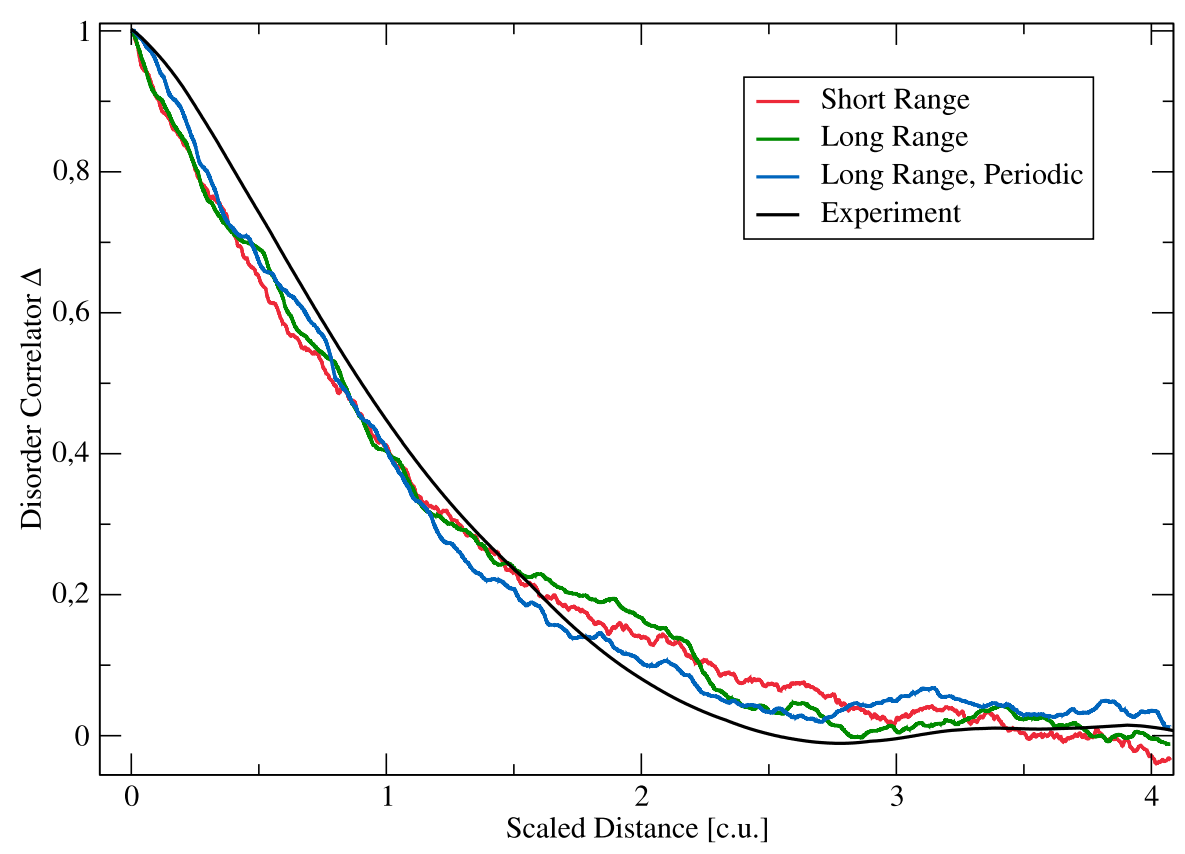

Fig. 5. Disorder correlator for simulations and experiments.

\section{Conclusions}

In this paper, we have briefly reviewed a particular experimental setup, in which one can study the dynamics of a crack front under the influence of disorder. One aspect to note here is the ease of sample preparation, we have often most used usual copy paper. The recent results show that experimental and numerical realizations of a line interface in random potential have the same characteristics. Earlier we 
have shown that the simulation model has the same energy, waiting time and velocity distributions [10], and studied the creep velocity in this system, using as a theoretical framework renormalization group arguments [4].

Our most recent results show that there are even more similarities in between the experiments and simulations. That is seen by analyzing the height timeseries using the autocorrelation, or the disorder correlator. Other aspects where the character of the elastic interactions become noticeable include as measure of correlation the third cumulant of the disorder correlator (see e.g. [2]).

\section{References}

1. D. S. Fisher, Physics Reports 301 (1998) 113.

2. P. Le Doussal, K. J. Wiese, S. Moulinet, and E. Rolley, Eur. Phys. Lett. 87 (2009) 56001.

3. L. I. Salminen, J. M. Pulakka, J. Rosti, M. J. Alava, and K. J. Niskanen, Eur. Phys. Lett. 73 (2006) 55 .

4. J. Koivisto, J. Rosti, and M. J. Alava, Phys. Rev. Lett. 99 (2007) 145504.

5. K. J. Måløy, S. Santucci, J. Schmittbuhl, and R. Toussaint, Phys. Rev. Lett. 96 (2006) 045501.

6. J. Heierli and M. Zaiser, Cold Reg. Sci. Tech. 52 (2008) 385.

7. E. Bouchaud, Journal of Physics: Condensed Matter 9 (1997) 4319.

8. A. B. Kolton, A. Rosso, and T. Giamarchi, Phys. Rev. Lett. 94 (2005) 047002.

9. P. Chauve, T. Giamarchi, and P. L. Doussal, Eur. Phys. Lett. 44 (1998) 110.

10. J. Rosti, J. Koivisto, X. Illa, P. Traversa, J.-R. Grasso, and M. J. Alava, Int. J. Frac. 151 (2008) 281.

11. L. I. Salminen, A. I. Tolvanen, and M. J. Alava, Phys. Rev. Lett. 89 (2002) 185503.

12. H. W. Haslach, Jr., Mech. Time-Dep. Mat. 4 (2000) 169.

13. R. Mark, C. Habeger, J. Borch, and M. Lyne (editors), Handbook of Physical Testing of Paper, (Marcel Dekker, New York).

14. M. Mustalahti, J. Rosti, J. Koivisto, and M. Alava, (submitted).

15. D. Coffin, The creep response of paper, 13th Fundamental Research Symposium, Cambridge, UK (2005).

16. J. Koivisto, X. Illa, and M. Alava, (submitted). 